\title{
Experiment on Jet Flow Control by SDBD Plasma Actuator
}

\author{
Jinlong $\mathrm{Li}^{\mathrm{a}}$, Xiuqian $\mathrm{Li}^{\mathrm{b}}$, Zheng Zhang ${ }^{\mathrm{c}}$, Xueke Che ${ }^{\mathrm{d}}$, Wansheng $\mathrm{Nie}^{\mathrm{e}}$ and \\ Zhi Zheng ${ }^{f}$ \\ Equipment Academy, Beijing, 101416, China \\ aklee111@mail.nwpu.edu.cn, bxiuqianli@hotmail.com, 'general_zz@163.com, dchedk@163.com, \\ enws1969@126.com, ${ }^{\mathrm{f}} \mathrm{dymzz1166@163.com}$
}

Keywords: jet flow, plasma actuator, surface dielectric barrier discharge, flow control.

\begin{abstract}
Single and twin jet flow control by surface dielectric barrier discharge plasma actuator is studied in this paper. The plasma actuator is placed on the exit of a rectangular nozzle and particle image velocimetry method is adopted to measure the jet flow velocity and conduct flow visualization. The test results prove that surface dielectric barrier discharge plasma actuator can disturb the boundary layer in the single jet, which changing the original symmetrical vortex structure, then the spreading performance can be improved. In the twin jet, the plasma actuator causes the shear layer instability, which can increase jet width significantly and show a mixing enhancement.
\end{abstract}

\section{Introduction}

Jets are important research content in fluid mechanics with many applications in industry, such as rocket, jet engine, air conditioning and chemical mixing technology [1]. It is important to improve jet flow properties such as mixing, spreading, and noise. Therefore, the control devices have been widely considered, which mainly divided into two categories: passive control and active control [2]. The passive control has been demonstrated have effect on jets with characteristics of simple structure and no external force, but the control effect is limited. Compared with passive control, active control has the characteristics of unsteady actuations and more control efficiency. Plasma actuator is an important active control method with characteristics of quick response and flexible position as well as simple structure [3], which has tremendous potential in restraining flow separation, controlling the boundary layer [4] and improving the engine efficiency [5]. Plasma actuator flow control was investigated by Che [6], Chen [7] and Cheng [8] et al. and they indicated that plasma actuator can suppress flow separation on airfoil and propeller. Samimy [9] and Crawle [10] et al. used localized arc filament plasma actuators to control high-speed jets, produce a greater reduction in acoustic radiation with a significant reduction jet potential core length. Bneard et al. [11] also demonstrated a mixing enhancement by plasma actuators placed along the lips of the diffuser. Kozato et al. [12] placed two opposite plasma actuators on the nozzle exit, they find that different actuation frequencies can change jet with and vortex morphology. However, there have been few studies on the surface dielectric barrier discharge(SDBD) plasma actuators application on jet flow control. Therefore, the characteristics of a single and a twin rectangular jet flow with SDBD plasma actuator placed on the nozzle exit are investigated via particle image velocimetry(PIV) method in this paper.

\section{Experimental Setup}

The experimental setup shown in Figure 1 consists of a cylindrical pressure vessel, control system, ac power supply, rectangular nozzle, and PIV system. The rectangular nozzle consists of two channels, with the nozzle width $27 \mathrm{~mm}$ and both channel height $3 \mathrm{~mm}(\mathrm{AR}=9)$. The channels are separated by quartz with a thickness of $1.5 \mathrm{~mm}$. Figure 1 (b) shows the detailed arrangement of SDBD plasma actuator placed on the exit of the nozzle. The actuator consists of exposed and encapsulated electrodes made of copper strips with width $5 \mathrm{~mm}$ and $9 \mathrm{~mm}$, respectively. The gap width between two electrodes is $0 \mathrm{~mm}$. And the dielectric barrier layer was made of Kapton with a thickness of $80 \mu \mathrm{m}$. The direction 
of the induced flow velocity is opposite to the direction of jet flow. The single jet flow control is studied when the upper channel working and the twin jet flow control is studied when two channels working.

The pressure vessel is made of stainless steel with an inner diameter $600 \mathrm{~mm}$ and a length $800 \mathrm{~mm}$. A quartz glass window with diameter $200 \mathrm{~mm}$ in the left wall is used to PIV camera measurements. The vessel pressure is monitored using a vacuum control cabinet with an accuracy of $100 \mathrm{~Pa}$ and pumping speed of $14 \mathrm{~L} / \mathrm{s}$. Air temperature and pressure in the vessel is $26^{\circ} \mathrm{C}$ and $78.8 \mathrm{kPa}$, respectively. The air inlet includes a smoke tank, valves and flowmeters, which are connected through the steel tubes.

The SDBD actuator is driven by a HFHV30-1 power supply[13] designed by the Institute of Electrical Engineering of the Chinese Academy of Sciences. The output voltage of the power supply is $\pm 30 \mathrm{kV}$ with a sinusoidal ac waveform. There are two working modes, continuous and impulse mode[14]. The output frequency of continuous mode is $1 \sim 50 \mathrm{kHz}$; the impulse frequency of the impulse mode is $10 \sim 3000 \mathrm{~Hz}$ and duty cycle is $10 \% \sim 90 \%$. In this paper, impulse mode is adopted.

The PIV system consists of a dual-head frequency doubled Nd:YAG laser, synchronous controllers, CCD camera, computers and image processing software. Light scattered from seeding droplets is observed with a $7.4 \mathrm{~lm}$ pixel dimension Kodak CCD camera having 2048×2048-pixel resolution. The camera is equipped with a Nikon $50 \mathrm{~mm} / \mathrm{F} 1.4$ lens and triggered in a two-frame burst mode at a repetition rate $5 \mathrm{~Hz}$. The ac voltage applied across the electrodes is measured by a high voltage probe (Agilent Model N2771B). Total discharge current is measured by a current probe (Pearson, Model 6595). All waveforms are recorded by a digital oscilloscope (Agilent Model DSO3024A)[15].

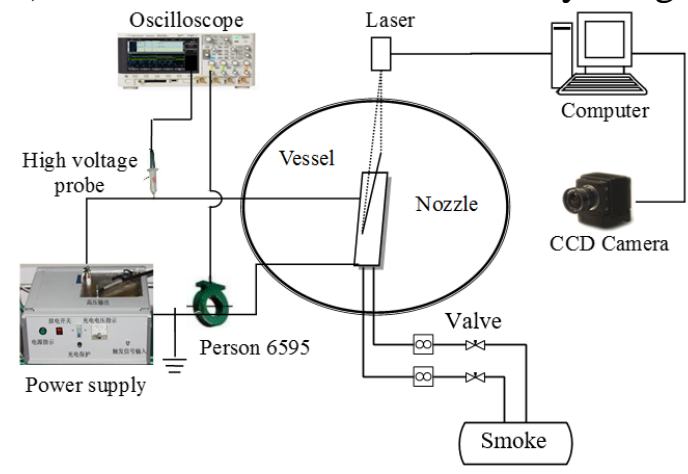

(a) Experimental setup

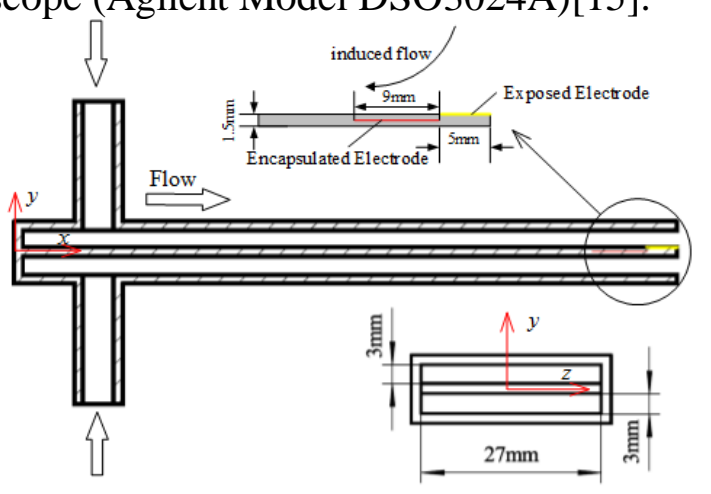

(b)Nozzle and SDBD plasma actuator in detail

Fig. 1 Schematics of the experimental system

\section{Results}

\subsection{Single Jet Flow Control}

Fig. 2 shows the comparison of the plasma actuator before and after the application on the single jet flow. The ac voltage magnitude, pulse frequency and duty cycle is kept at $5 \mathrm{kV}, 50 \mathrm{~Hz}$ and $50 \%$, respectively. The flow patterns are shown in Fig.2(a) and 2(b), in which jet velocity is $6.2 \mathrm{~m} / \mathrm{s}$ and 11 $\mathrm{m} / \mathrm{s}$, respectively. At the same, Fig. 2(c) and 2(d) are the velocity contours which can prove more information for analysis. When the plasma actuator off, the single jet flow can produce symmetrical vortex structure, which is the same as result obtained in literature[12]. When the plasma actuator on, a reverse jet can be produced in the boundary layer, so that it can disturb the jet flow and then symmetrical vortex structure would be destroyed. Because of the interference among vortex with the jet development, the spreading performance can be improved by plasma actuator. When the jet velocity increases, with the plasma actuator on, the jet initial section decreases and the width of the jet main section decreases slightly. 


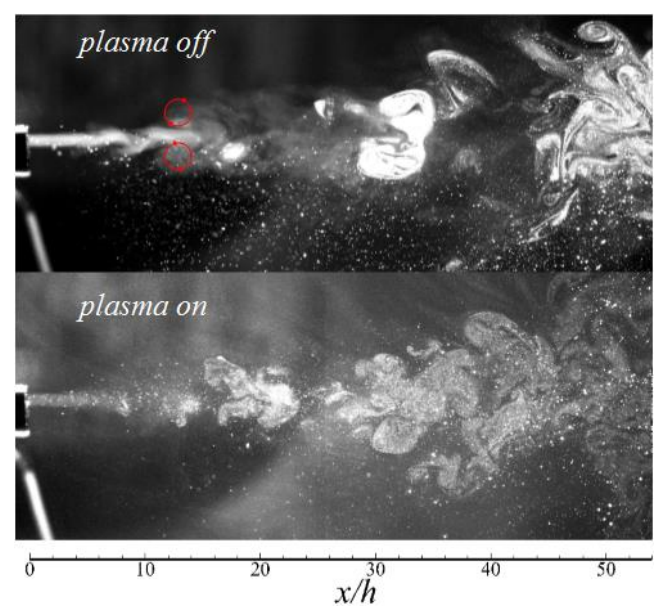

(a) $6.2 \mathrm{~m} / \mathrm{s}$ jet flow images
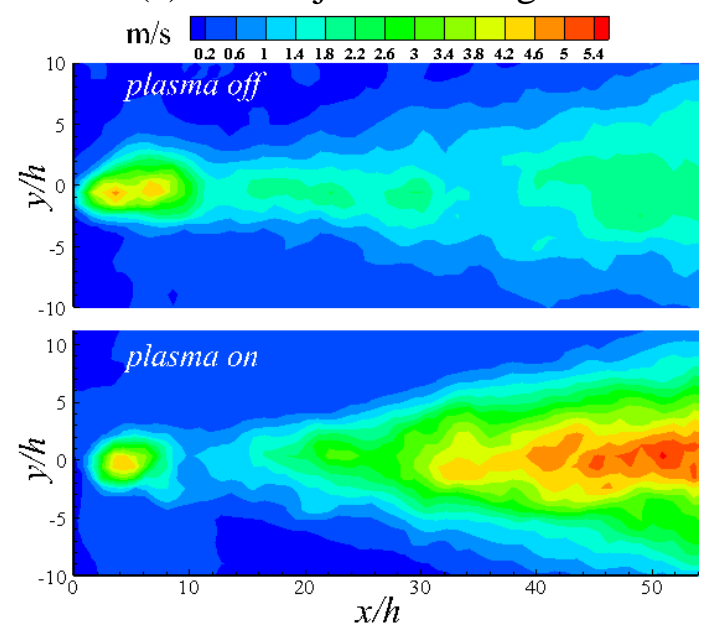

(c) $6.2 \mathrm{~m} / \mathrm{s}$ jet flow velocity contours

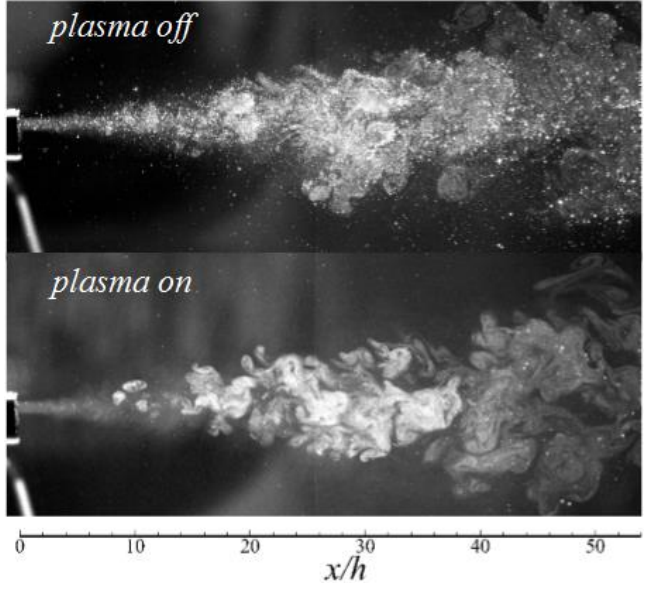

(b) $11 \mathrm{~m} / \mathrm{s}$ jet flow images
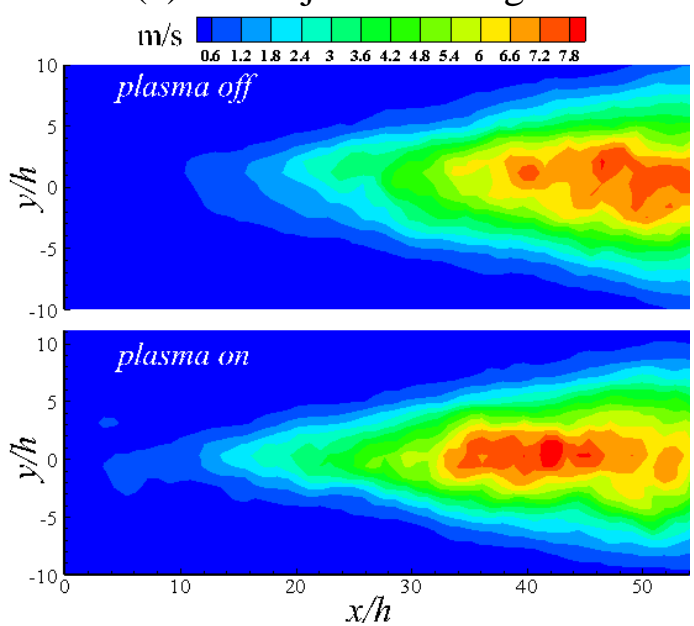

(d) $11 \mathrm{~m} / \mathrm{s}$ jet flow velocity contours

Fig. 2 Single jet flow images and velocity contours

\subsection{Twin Jet Flow Control}

Fig. 3 shows the comparison of the plasma actuator before and after the application on the twin jet flow. When the plasma actuator off, two jets become mixing approximately at $x / h=4$, then develop into one jet. When the plasma actuator on, with the same actuation parameter, the mixing point change insignificantly. It can be found that the jet width become lager, and vortex structure collapses easily. In this condition, the plasma actuator causes the shear layer instability, which lead two jets show a mixing enhancement significantly. The mixing enhancement is obtained under this specific actuation parameter, so the future work would be concentrated on the different jet flow control with changing actuation parameter, actuators position and velocity ratio, and exploring the SDBD plasma flow control mechanism.

\section{Conclusion}

In this paper, SDBD plasma actuator is placed on the exit of a rectangular nozzle to investigate jet flow control experimentally. PIV method is used to conduct flow visualization and measure the velocity field. The primary conclusions are as follows:

1) SDBD plasma actuator has effective control on both single and twin jet.

2) In the single jets with different velocity, SDBD plasma actuator can disturb the boundary layer and change the symmetrical vortex structure, which can change the spread of the jet, while the jet width changes insignificantly.

3) In the twin jet, SDBD plasma actuator can causes the shear layer instability and vortex structure collapse easily, which can enhance the twin jet flow mixing property; the jet width becomes lager with mixing point changes insignificantly. 


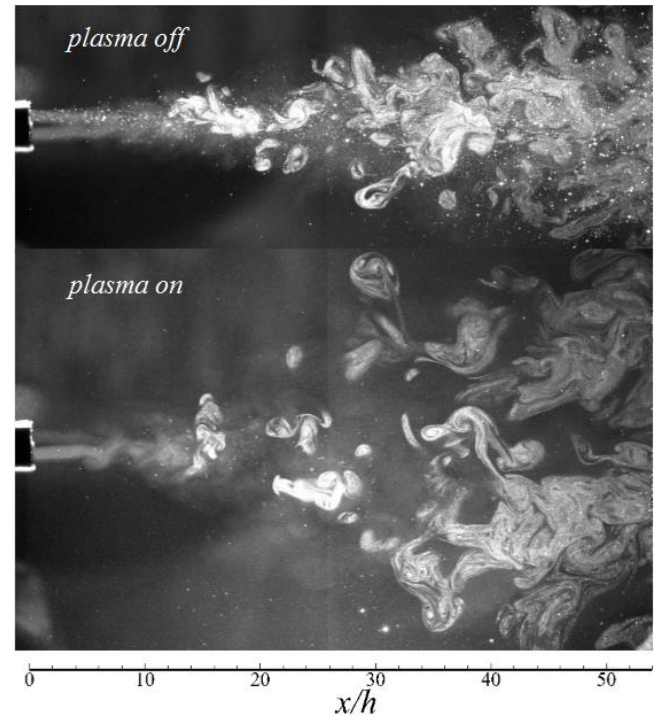

(a)Twin jet flow images

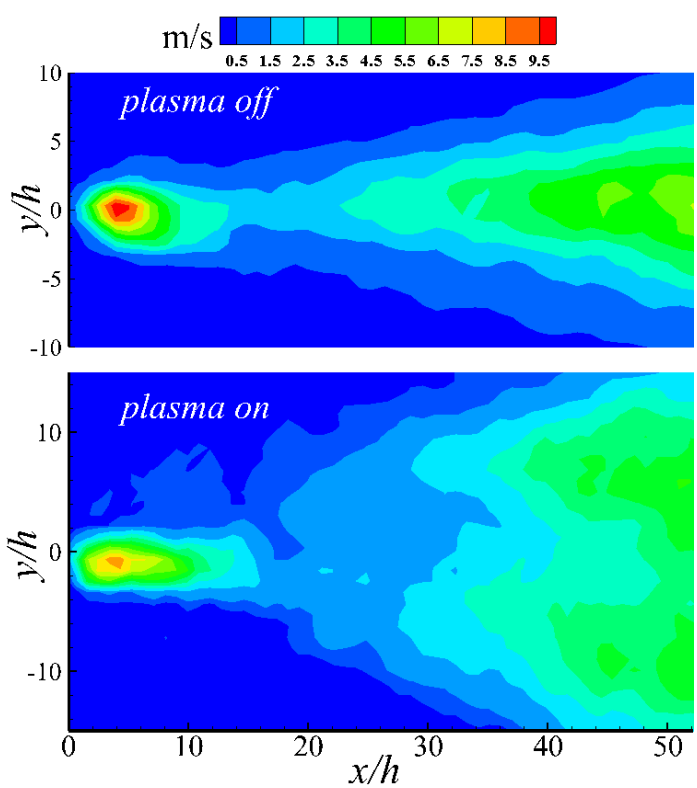

(b) Twin jet flow velocity contours

Fig. 3 Twin jet flow images and velocity contours

\section{References}

[1]. Ping J, Basic theory and application of jet [M]. Beijing, China Astronautic Publishing House, 1995, p. 1-5.

[2]. Gad-el-Hak, Mohamed. Flow control: passive, active, and reactive flow management [M]. Cambridge University Press, 2000, p. 2-10.

[3]. Wang J J, Choi K S, Feng L H, et al. Recent developments in DBD plasma flow control [J]. Progress in Aerospace Sciences, Vol. 62(2013) No. 4, p. 52-78.

[4]. Nie W S, Cheng Y F, and Che X K. A review on dielectric barrier discharge plasma flow control, Advances in Mechanics, Vol. 42(2012) No. 6, p. 722-734.

[5]. Wu Y, Li Y H. Progress in research of plasma-assisted flow Control, ignition and combustion [J]. High Voltage Engineering, Vol. 40(2014) No. 7, p. 2024-2038.

[6]. Che X K, Nie W S, Tian X H, et al. Similarity criteria of surface dielectric barrier discharge plasma induced flow-field and its application [J]. High Voltage Engineering, Vol. 42(2016) No. 3, p. 769-774.

[7]. Chen Q Y, Tian X H, Jiang J W, et al. Experiment on the performance of propeller plasma flow control [J]. Journal of Aerospace Power, Vol. 31(2016) No. 5, p. 1205-1211.

[8]. Cheng Y F, Che X K, Nie W S. Numerical Study on Propeller Flow-Separation Control by DBD-Plasma Aerodynamic Actuation [J]. IEEE Transactions on Plasma Science, Vol. 41(2013) No. 4, p. 892-898.

[9]. Samimy M, Kim J H, Adamovich I, et al. Active Control of High Speed and High Reynolds Number Free Jets Using Plasma Actuators [J]. Journal of Fluid Mechanics, Vol. 578(2007), p. 2024-2038.

[10]. Crawley M, Kearneyfischer M, Mo S. Control of a Supersonic Rectangular Jet Using Plasma Actuators [R]. AIAA Paper: 2012-2211.

[11]. Benard N, Balcon N, Touchard G, et al. Control of diffuser jet flow: turbulent kinetic energy and jet spreading enhancements assisted by a non-thermal plasma discharge [J]. Experiments in Fluids, Vol. 45(2014) No. 2, p. 333-355. 
[12]. Kozato Y, Kikuchi S, Imao S, et al. Flow control of a rectangular jet by DBD plasma actuators [J]. International Journal of Heat \& Fluid Flow, Vol. 62(2016), p. 33-43.

[13]. Shao T, Wang L, Zhang C, et al. A Compact Microsecond-Pulse Generator Used for Surface Dielectric Barrier Discharges [J]. IEEE Transactions on Plasma Science, Vol. 44(2016) No. 10, p. 2072-2078.

[14]. Tian X M, Tian X H, Che X K, et al. Experimental study on unsteady plasma induced flow field [J]. High Voltage Engineering, Vol. 41(2015) No. 12, p. 4060-4065.

[15]. Che X K, Nie W S, Shao T, et al. Study of flow fields induced by surface dielectric barrier discharge actuator in low-pressure air [J]. Physics of Plasmas, Vol. 21(2014) No. 4, p. 289-318. 